\title{
Article
}

\section{Pulsation in the white dwarf HE 1017- 1352: confirmation of the class of hot DAV stars}

Romero, Alejandra D, Amaral, L Antunes, Kepler, S O, Fraga, L, Kurtz, Donald Wayne and Shibahashi, H

Available at http://clok.uclan.ac.uk/33673/

Romero, Alejandra D, Amaral, L Antunes, Kepler, S O, Fraga, L, Kurtz, Donald Wayne ORCID: 0000-0002-1015-3268 and Shibahashi, H (2020) Pulsation in the white dwarf HE 1017-1352: confirmation of the class of hot DAV stars.

Monthly Notices of the Royal Astronomical Society: Letters, 497 (1). L24-L29.

It is advisable to refer to the publisher's version if you intend to cite from the work. http://dx.doi.org/10.1093/mnrasl/slaa106

For more information about UCLan's research in this area go to http://www.uclan.ac.uk/researchgroups/ and search for <name of research Group>.

For information about Research generally at UCLan please go to http://www.uclan.ac.uk/research/

All outputs in CLoK are protected by Intellectual Property Rights law, including Copyright law. Copyright, IPR and Moral Rights for the works on this site are retained by the individual authors and/or other copyright owners. Terms and conditions for use of this material are defined in the policies page.

\section{CLoK}

Central Lancashire online Knowledge www.clok.uclan.ac.uk

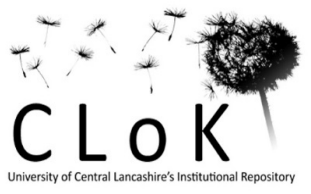




\title{
Pulsation in the white dwarf HE 1017-1352: confirmation of the class of hot DAV stars
}

\author{
Alejandra D. Romero ${ }^{\oplus}{ }^{1 \star}$ L. Antunes Amaral, ${ }^{1}$ S. O. Kepler ${ }^{\oplus},{ }^{1}$ L. Fraga, ${ }^{2}$ \\ D. Kurtz ${ }^{\oplus 3,4}$ and H. Shibahashi ${ }^{5}$ \\ ${ }^{1}$ Instituto de Física, Universidade Federal do Rio Grande do Sul, 91501-900 Porto Alegre, RS, Brazil \\ ${ }^{2}$ Laboratório Nacional de Astrofísica (LNA), MCTIC, 37504-364 Itajubá, MG, Brazil \\ ${ }^{3}$ Department of Physics, North-West University, Dr Albert Luthuli Drive, Mahikeng 2735, South Africa \\ ${ }^{4}$ Jeremiah Horrocks Institute, University of Central Lancashire, Preston PR1 2HE, UK \\ ${ }^{5}$ Department of Astronomy, School of Science, University of Tokyo, Tokyo 113-0033, Japan
}

Accepted 2020 May 28. Received 2020 May 28; in original form 2020 April 9

\begin{abstract}
We report the detection of periodic variations on the $T_{\text {eff }} \simeq 32000 \mathrm{~K} \mathrm{DA}$ white dwarf star HE 1017-1352. We obtained time series photometry using the 4.1-m Southern Astrophysical Research telescope on three separate nights for a total of $16.8 \mathrm{~h}$. From the frequency analysis, we found four periods of $605,556,508$, and $869 \mathrm{~s}$ with significant amplitudes above the 1/1000 false alarm probability detection limit. The detected modes are compatible with low harmonic degree g-mode non-radial pulsations with radial order higher than $\sim 9$. This detection confirms the pulsation nature of HE 1017-1352 and thus the existence of the new pulsating class of hot DA white dwarf stars. In addition, we detect a long period of $1.52 \mathrm{~h}$, compatible with a rotation period of DA white dwarf stars.
\end{abstract}

Key words: stars: interiors - stars: variables: general - white dwarfs.

\section{INTRODUCTION}

Pulsating white dwarf stars can be found in several temperature ranges along the white dwarf evolutionary cooling tracks. The excitation mechanisms are a combination of an opacity bump due to partial ionization of the main element in the outer layers $(\kappa$-mechanism) and the effect of a small value of the adiabatic exponent $\Gamma_{3}-1$ in the ionization zone, preventing a large increase in temperature upon compression $(\gamma$-mechanism). Since ionization occurs at different temperatures for different elements, pulsation instabilities are expected at different effective temperatures. For instance, the excitation mechanism for helium atmosphere white dwarfs (DB) is consistent with the $\kappa-\gamma$ mechanism due to the first helium ionization (He I-He II), as predicted by Winget et al. (1982). The pulsating DB white dwarf stars are found in the temperature range of $25000 \mathrm{~K} \lesssim T_{\text {eff }} \lesssim 30000 \mathrm{~K}$. Hydrogen atmosphere white dwarf stars (DA) near the blue edge of the DB instability strip could develop pulsation, if the hydrogen layer is thin enough. This hypothesis was proposed by Shibahashi $(2005,2007)$ to predict the existence of pulsation in 'hot DA' white dwarf stars, with effective temperatures around $\sim 30000 \mathrm{~K}{ }^{1}$

\footnotetext{
^E-mail: aleromero82@gmail.com, alejandra.romero@ufrgs.br

${ }^{1}$ Winget et al. (1982) found that the He partial ionization could also excite pulsations in DA white dwarfs with $M_{\mathrm{H}} / M_{*}<10^{-10}$ and $T_{\text {eff }} \sim 19000 \mathrm{~K}$.
}

Shibahashi $(2005,2007)$ found that around $30000 \mathrm{~K}$ the atmosphere of a white dwarf is superadiabatic and convectively stabilized by a chemical composition gradient ( $\mu$-gradient). Gravitational settling is very efficient for white dwarfs, leading to a stratified structure, where the lighter, cooler hydrogen is floating on top of the heavier helium layer. Under this condition, he found that the radiative heat exchange leads to an asymmetry in g-mode oscillatory motion such that the oscillating elements overshoot their equilibrium positions with increasing velocity. A linear local stability analysis based on the dispersion relation led him to predict that $g$ modes should be excited in DA stars at the blue edge of the DBV instability strip, pulsating in higher degree modes, but with some $\ell<3$ modes excited. In the models, this could only occur for thin hydrogen envelopes of about $10^{-12} \mathrm{M}_{\odot}$. However, the mass of the hydrogen envelope must be larger than about $10^{-14} \mathrm{M}_{\odot}$, otherwise it will be diluted into the more massive helium layer due to convective mixing, and the star will no longer be a DA white dwarf for $T_{\text {eff }} \sim 30000 \mathrm{~K}$ (Fontaine \& Wesemael 1987).

Following the predictions of Shibahashi $(2005,2007)$, Kurtz et al. (2008) performed a search for pulsations in a sample of seven DA white dwarf stars with spectroscopic effective temperatures in the effective temperature range of 31 200-29 500 K. They reported two possible variable hot DAV stars: SDSS J010415.99+144857.4 and SDSS J023520.02-093465.3, with periods of 750 and $159 \mathrm{~s}$, respectively. Later, Kurtz et al. (2013) found a third candidate, HE 1017-1352 (WD 1017-138), showing a period of $615 \mathrm{~s}$, based 


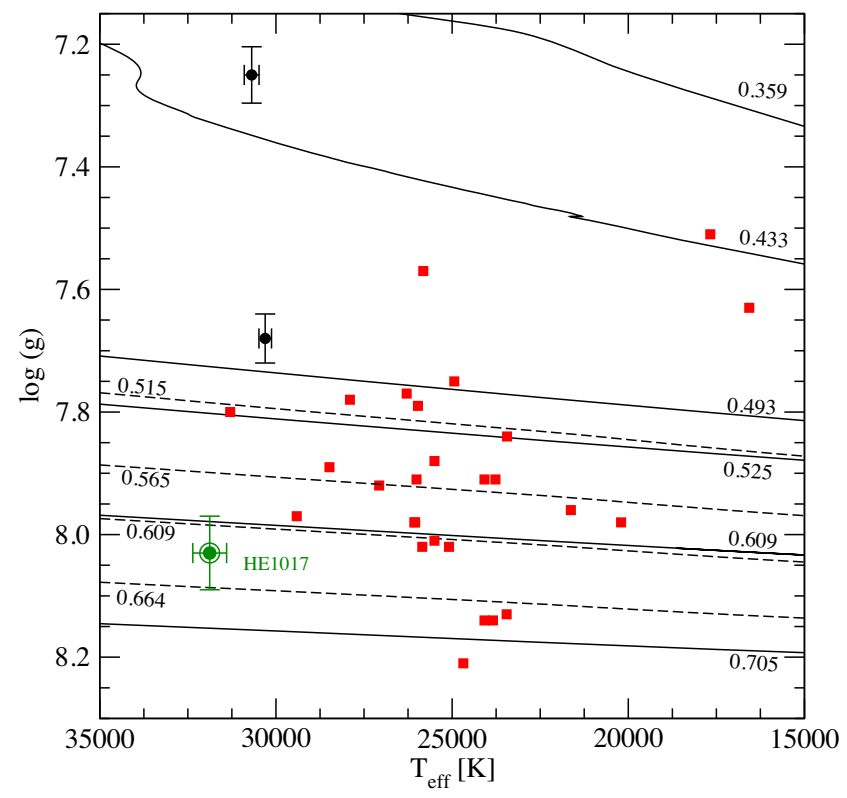

Figure 1. The position of the three hot DAV candidates and DBV white dwarf stars in the $T_{\text {eff }}-\log g$ plane. Hot DAV candidates from Kurtz et al. (2008) are depicted with black circles, while HE 1017-1352 is depicted with a green circle. Known DBV stars are depicted with red squares. Some cooling tracks with thin hydrogen envelope (full line; Romero et al. 2019a) and pure helium atmospheres (dashed line; Althaus et al. 2009) are included for masses from 0.493 to $0.705 \mathrm{M}_{\odot}$. Two low-mass sequences with thick hydrogen envelopes, with stellar masses of 0.359 and $0.433 \mathrm{M}_{\odot}$, are also included (Romero \& Istrate 2019). Atmospheric parameters for HE 1017-1352 are taken from Gianninas et al. (2011), and for the other two hot DAV candidates are taken from Kepler et al. (2016).

on two independent 2.3 and 2.1-h photometric time series obtained with the 4.2-m William Herschel Telescope (WHT).

The hot DAV stars presented by Kurtz et al. $(2008,2013)$ are depicted in Fig. 1 in the $T_{\text {eff }}-\log g$ plane. The target of this study, HE 1017-1352, is depicted by a green circle, with atmospheric parameters of $T_{\text {eff }}=32000 \pm 500 \mathrm{~K}$ and $\log g=8.03 \pm 0.06$, taken from Gianninas, Bergeron \& Ruiz (2011). The two hot DAV candidates presented in Kurtz et al. (2008) are depicted with black circles (see Kepler et al. 2016 for details). For comparison, we plot 27 of the 46 known pulsating DB white dwarf stars with red squares (Koester \& Kepler 2015; Vanderbosch, Winget \& Winget 2018). Theoretical cooling sequences for thin hydrogen and pure helium envelope white dwarf models are included for stellar masses from 0.493 to $0.705 \mathrm{M}_{\odot}$, along with low-mass hydrogen envelope sequences.

Even after the works of Kurtz et al. (2008, 2013), confirmation of variability in hot DA white dwarf stars is still needed to establish the existence of a new class of pulsating white dwarf stars. In this work, we perform follow-up observations on HE 1017-1352 with the 4.1-m Southern Astrophysical Research (SOAR) telescope. We present our data analysis and results in Section 2. In addition, we present data from the Transiting Exoplanet Survey Satellite (TESS) and compared both with the original data from Kurtz et al. (2013). Concluding remarks are presented in Section 3.

\section{THE SUSPECT: HE 1017-1352}

HE 1017-1352 $\left(\alpha_{2000}=10: 19: 52.36, \delta_{2000}=-14: 07: 34.26\right)$ is a $V=14.6$ DA white dwarf star with an effective temperature
Table 1. Journal of observations for HE 1017-1352 with the SOAR telescope. $\Delta t$ is the length of each observing run and $t_{\exp }$ is the exposure time of each exposure.

\begin{tabular}{lcc}
\hline Run start (UT) & $t_{\exp }(\mathrm{s})$ & $\Delta t(\mathrm{~h})$ \\
\hline 2020 January 22, 04:44:23.35 & 5 & 3.76 \\
2020 March 7, 01:16:48.89 & 5 & 6.07 \\
2020 March 8, 01:08:33.73 & 5 & 6.93 \\
\hline
\end{tabular}

close to the blue edge of the DBV instability strip, as shown in Fig. 1. Gianninas et al. (2011) found atmospheric parameters of $T_{\text {eff }}=32000 \pm 500 \mathrm{~K}$ and $\log g=8.03 \pm 0.06$ (cgs units), using atmosphere models with a mixing length of $0.8 H_{\mathrm{p}}$, where $H_{\mathrm{p}}$ denotes the pressure scale height, while Koester et al. (2009) found values of $T_{\text {eff }}=31800 \pm 30 \mathrm{~K}$ and $\log g=7.840 \pm 0.006$, using models with $0.6 H_{\mathrm{p}}$. Since the models do not have significant surface convection, the value of $\alpha$ is not dominant. Note that the quoted uncertainties correspond to the statistical uncertainties of the fitting procedure and are probably underestimated. These two determinations are in agreement within $0.2 \sigma$ in $T_{\text {eff }}$ and $3.2 \sigma$ in $\log g$, but most importantly they agree that HE 1017-1352 is a hot DA white dwarf star.

From the spectroscopic parameters, we computed the stellar mass using the white dwarf evolutionary sequences from Romero et al. (2019a). For theoretical sequences with canonical hydrogen envelopes - those obtained from single stellar evolution computations - we obtained a stellar mass of $0.661 \pm 0.030 \mathrm{M}_{\odot}$ considering the spectroscopic values from Gianninas et al. (2011) and $0.566 \pm 0.003 \mathrm{M}_{\odot}$ for those obtained by Koester et al. (2009). The stellar mass is reduced to $0.636 \pm 0.031$ and $0.541 \pm 0.003 \mathrm{M}_{\odot}$, respectively, if we consider theoretical sequences with thin hydrogen envelopes $\left(M_{\mathrm{H}} \sim 10^{-9.5} M_{*}\right)$. We can also estimate the stellar mass from the parallax and magnitudes from the Gaia mission data release 2, independently from spectroscopy (see Romero et al. 2019b, for details). We employ thin hydrogen atmosphere models from the Montreal group ${ }^{2}$ (Bergeron, Wesemael \& Beauchamp 1995) for Gaia magnitudes to transform absolute magnitude and colour into stellar mass and effective temperature. For HE 1017-1352, we find that $\pi=9.34 \pm 0.14$ mas, $G=14.559$, and $G_{b_{p}}-G_{r_{p}}=-0.439$, lead to a stellar mass of $0.421 \pm 0.008 \mathrm{M}_{\odot}, 22-33$ percent lower than the value obtained from spectroscopy. The photometric effective temperature in this case is around $31200 \mathrm{~K}$.

\subsection{SOAR data: frequency analysis and discussion}

For the observations of HE 1017-1352, we employed the Goodman spectrograph in image mode on the 4.1-m SOAR telescope. We used the CCD binned $2 \times 2$ and a region of interest of $800 \times 800$ pixels, yielding a plate scale of 0.30 arcsec per pixel and a field of view of $4 \times 4$ arcmin. This set-up yielded a readout time of $7 \mathrm{~s}$. All observations were obtained with a red blocking filter S8612 (transmitting $\lambda=330-620 \mathrm{~nm}$ ) to decrease sky contamination. We observed the target for three nights in the beginning of 2020 , for a total of $16.76 \mathrm{~h}$. The journal of observations is presented in Table 1 .

We reduced the data with the IRAF software and performed aperture photometry with the DAOPHOT task. We used differential

\footnotetext{
${ }^{2}$ http://www.astro.umontreal.ca/ bergeron/CoolingModels/
} 
photometry to minimize effects of sky and transparency fluctuations. We tested all the comparison stars in the field; from the three brightest stars, we used the light curve with the best signal-to-noise ratio. To look for periodicities in the light curves, we calculated a discrete Fourier transform (FT) using the period04 software (Lenz \& Breger 2004).

The FTs for each night are presented in Fig. 2, and the detected frequencies are listed in Table 2. The detection limit (dashed line) corresponds to the $1 / 1000$ false alarm probability (FAP), where any peak with amplitude above this value has 0.1 per cent probability of being a false detection due to noise. The FAP is calculated by shuffling the fluxes in the light curve while keeping the same time sampling, and computing the FT of the randomized data. This procedure is repeated $N / 2$ times, where $N$ is the number of points in the light curve. For each run, we compute the maximum amplitude of the FT. From the distribution of maxima, we take the 0.999 percentile to be the detection limit. The internal uncertainties in frequency and amplitude were computed using a Monte Carlo method with 1000 simulations with period 04 , while uncertainties in the periods were obtained through error propagation.

For the three nights, a peak with long period at $\sim 5400 \mathrm{~s}(\sim 1.5 \mathrm{~h})$ is present with significant amplitude. This 1.5 -h period is compatible with a rotation period of DA white dwarf stars, in particular for ZZ Ceti stars near the blue edge of the instability strip (Kepler et al. 2017). Note that this period is coherent over the three nights of observation. The peaks at low frequencies, for periods longer than $1.5 \mathrm{~h}$, are a consequence of the interference of Earth atmosphere and they are also observed in the light curve of the comparison stars. In addition, our observations are not long enough to resolve such long periods. The peak corresponding to a period of $\sim 606 \mathrm{~s}$ is dominant in the first night (top panel of Fig. 2), for which its harmonic is also present at $302.1 \mathrm{~s}$. For the second and third nights (middle and bottom panels), this mode decreased in amplitude, while the peak with a period of $\sim 557 \mathrm{~s}$ increased. As a consequence of the energy loss, the peak with $302.1 \mathrm{~s}$, corresponding to the harmonic of the $\sim 606 \mathrm{~s}$ peak, disappears. In addition, the peak corresponding to a period of $\sim 508 \mathrm{~s}$ increased its amplitude above the FAP (1/1000) detection limit. As expected, we can better resolve the three frequencies in the second and third nights, since the observation time is longer than the beat period of $\sim 4 \mathrm{~h}$ (see Table 1).

Finally, we computed the FT of the concatenation of the three nights of observations with the SOAR telescope, with $16.76 \mathrm{~h}$ of data, after correcting the timings to barycentric dynamical time. The result is presented in the middle panel of Fig. 3, and the list of detected frequencies, amplitudes, and periods is presented in the first three columns of Table 3. We find three modes with periods of 508.121, 556.802, and $605.310 \mathrm{~s}$ with significant amplitudes above the FAP (1/1000) detection limit. At lower frequencies, a low-amplitude peak is also present in the FT, corresponding to a period of $869.358 \mathrm{~s}$. These periods are consistent with low harmonic degree g modes in white dwarf stars. If we consider that the periods of 508.121, 556.802, and $605.310 \mathrm{~s}$ correspond to modes with consecutive radial order $k$, we can estimate the forward period spacing as $\Delta \Pi_{k}=\Pi_{k+1}-\Pi_{k} \sim 48-49 \mathrm{~s}$. This value is in agreement with the expected value of the asymptotic period spacing for $\sim 0.5$ $0.6 \mathrm{M}_{\odot}$ white dwarf stars (Tassoul 1980). In summary, we found four peaks in the FT for HE 1017-1352 consistent with pulsations.

At low frequencies, the peak with the largest amplitude shows a period of $1.52 \mathrm{~h}$, which we propose as a rotation period. However, it is possible that low-frequency peaks are subharmonics of shorter periods. While linear combinations tend to have smaller amplitudes than the parent modes, Kurtz et al. (2015) showed that it is theoretically possible for combination frequencies to have amplitudes greater than the base frequency amplitudes, and that this is observed in some $\gamma$ Dor and slowly pulsating B stars. None the less, the frequency corresponding to $1.52 \mathrm{~h}$ is not compatible with any linear combination of the three shorter detected periods, within the uncertainties.

We searched for a preliminary seismic solution, considering the periods obtained from the SOAR observations (see column 3 of Table 3). We first computed adiabatic pulsations for a white dwarf model characterized by $0.632 \mathrm{M}_{\odot}, T_{\text {eff }}=31870 \mathrm{~K}$, and $\log \left(M_{\mathrm{H}} / \mathrm{M}_{\odot}\right)=-12.6$ (see Romero et al. 2012, for details), following the spectroscopic parameters obtained from Gianninas et al. (2011). For this model, we can fit the four observed periods $508.121,556.802,605.310$, and 869.358 s, with $\ell=1$ theoretical modes with periods of 507.8, 556.1, 619.5, and 851.6 s, corresponding to radial numbers $k=13,14,16$, and 24 , respectively. In addition, we also considered a $\mathrm{C} / \mathrm{O}$ core white dwarf model with $0.423 \mathrm{M}_{\odot}, T_{\text {eff }}=31200 \mathrm{~K}$, and $\log \left(M_{\mathrm{H}} / \mathrm{M}_{\odot}\right)=-12.6$, which matches the photometric stellar mass and effective temperature based on Gaia data. For this model, we can fit the four observed periods with theoretical periods of $518.9,547.4,601.5$, and $882.8 \mathrm{~s}$, corresponding to radial numbers $k=10,11,12$, and 19 .

\subsection{Comparison with WHT and TESS data}

As mentioned before, photometric variability was reported for HE 1017-1352 from observations performed by Kurtz et al. (2013). They observed this star using the WHT on two nights for a total of $4.4 \mathrm{~h}$ and reported a period at $624 \mathrm{~s}$. For comparison purposes, we recomputed the FT for the original data obtained by Kurtz et al. (2013), shown in the bottom panel of Fig. 3, after correcting the timings to barycentric dynamical time. From our analysis, we found a period at $614 \mathrm{~s}$. The internal uncertainties for WHT data are underestimated due to beating. Considering that the uncertainty related to the FWHM of the peak in the WHT observations is $280 \mu \mathrm{Hz}$, or $105 \mathrm{~s}$ (see inset plot in bottom panel of Fig. 3), the period at $614 \mathrm{~s}$ is in agreement with the peak at $605 \mathrm{~s}$ obtained from SOAR data. In addition, a second peak at 572 s seems to be also present in the data from the WHT. However, due to the low resolution in frequency of the data it is not possible to separate it from the main peak. The list of frequencies, amplitudes, and periods is presented in Table 3. Because the WHT data are composed by two chunks of $\sim 2 \mathrm{~h}$ duration, separated by $3 \mathrm{~d}$, there is a strong correlation between the peaks and a single FAP value is not reliable, leading to an underestimated detection limit in this case. The additional peaks above the FAP (1/1000) limit that are present in the FT for the WHT data are part of the background noise and we only consider the two peaks listed in Table 3 to be real periodicities.

HE 1017-1352 was also observed by the TESS (TIC 307982318) in sector 9 for a 24-d run (2019 February $28 T 17: 17: 11.55$ to 2019 March 25 T23:30:40.318) with a 2 min cadence. The light curve was downloaded from Mikulski Archive for Space Telescopes (MAST). ${ }^{3}$ We have used the PDCSAP flux that removes common instrumental trends giving a pre-search data conditioning (PDC). Additionally, we have performed a sigma clipping for any data more than $5 \sigma$ away from the mean value of the light curve. The FT for the TESS data is shown in the top panel of Fig. 3 (see also Table 3). As can be seen from this figure, the TESS data are not conclusive on the detection of pulsations since there is no peak with amplitude above

\footnotetext{
${ }^{3}$ https://archive.stsci.edu/
} 


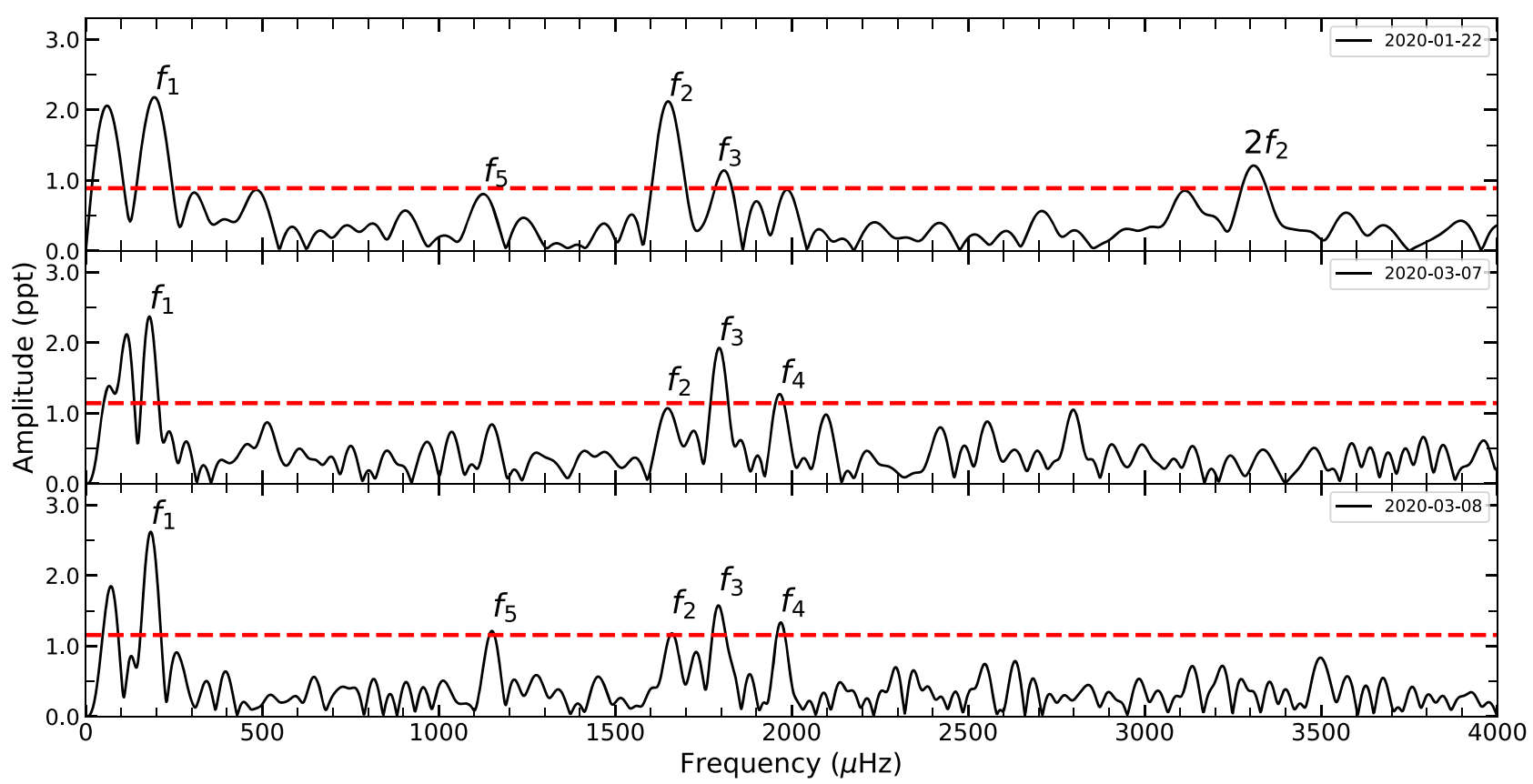

Figure 2. FT for HE 1017-1352 for the SOAR observations for each of the three nights listed in Table 1. The 1/1000 FAP detection limit was computed using random shuffling of the data. The identification for each peak is indicated (see Table 2). The FT corresponding to the concatenation of the three nights is depicted in the middle panel of Fig. 3.

Table 2. Detected frequencies for HE 1017-1352 from the observations performed with SOAR. We list the frequency, amplitude, and period for each peak in the FT depicted in Fig. 2 for each night. The quoted uncertainties correspond to the internal uncertainties. The value for the detection limit with 99.9 per cent confidence is indicated for each night in the first row. The last column shows the identification of the mode. The list of frequencies for the complete light curve, considering the concatenation of the three nights, is presented in Table 3 .

\begin{tabular}{|c|c|c|c|c|c|c|c|c|c|}
\hline $\begin{array}{l}\text { Frequency } \\
(\mu \mathrm{Hz})\end{array}$ & $\begin{array}{l}\text { Amplitude } \\
\text { (ppt) }\end{array}$ & $\Pi(\mathrm{s})$ & $\begin{array}{l}\text { Frequency } \\
\quad(\mu \mathrm{Hz})\end{array}$ & $\begin{array}{l}\text { Amplitude } \\
\text { (ppt) }\end{array}$ & $\Pi(\mathrm{s})$ & $\begin{array}{c}\text { Frequency } \\
\qquad(\mu \mathrm{Hz})\end{array}$ & $\begin{array}{l}\text { Amplitude } \\
\text { (ppt) }\end{array}$ & $\Pi(\mathrm{s})$ & ID \\
\hline \multicolumn{3}{|c|}{2020 January $22(0.9$ ppt $)$} & \multicolumn{3}{|c|}{$2020 \operatorname{March} 7$ (1.1 ppt) } & \multicolumn{3}{|c|}{2020 March 8 (1.2 ppt) } & \\
\hline $195 \pm 5$ & $2.2 \pm 0.2$ & $5107 \pm 130$ & $180 \pm 3$ & $2.4 \pm 0.4$ & $5539 \pm 92$ & $184 \pm 2$ & $2.6 \pm 0.3$ & $5424 \pm 59$ & $f_{1}$ \\
\hline $1651 \pm 4$ & $2.1 \pm 0.2$ & $605 \pm 1$ & $1645 \pm 6$ & $1.2 \pm 0.2$ & $607 \pm 2$ & $1659 \pm 4$ & $1.3 \pm 0.2$ & $602 \pm 1$ & $f_{2}$ \\
\hline $1787 \pm 7$ & $1.0 \pm 0.2$ & $559 \pm 2$ & $1793 \pm 4$ & $1.9 \pm 0.3$ & $557 \pm 1$ & $1793 \pm 3$ & $1.6 \pm 0.3$ & $557.6 \pm 0.9$ & $f_{3}$ \\
\hline $3309 \pm 5$ & $1.3 \pm 0.2$ & $302.1 \pm 0.5$ & - & - & - & - & - & - & $2 f_{2}$ \\
\hline- & - & - & $1967 \pm 7$ & $1.2 \pm 0.3$ & $508 \pm 2$ & $1967 \pm 4$ & $1.3 \pm 0.2$ & $508 \pm 1$ & $f_{4}$ \\
\hline $1126 \pm 9$ & $0.8 \pm 0.1$ & $887 \pm 7$ & - & - & - & $1152 \pm 5$ & $1.2 \pm 0.2$ & $867 \pm 4$ & $f_{5}$ \\
\hline
\end{tabular}

its FAP (1/1000) detection limit. For white dwarf stars as hot as HE 1017-1352, the flux curve peaks at UV wavelengths. Unlike the S816 filter used in the observations with SOAR telescope, the TESS bandpass is equivalent to a red filter, covering a wavelength range of $600-1000 \mathrm{~nm}$, where the photometric amplitude for hot stars is very low compared to that at bluer wavelengths (e.g. Robinson, Kepler \& Nather 1982). For $T_{\text {eff }} \sim 12000 \mathrm{~K} \mathrm{DAV} \mathrm{white} \mathrm{dwarf} \mathrm{stars,}$ it reduces their amplitude by about 63 per cent (Bognár et al. 2020). However, the largest peak occurs at $605.73 \mathrm{~s}$. In addition, the peak at $5482.92 \mathrm{~s}$, or $1.52 \mathrm{~h}$, is present, in agreement with the long period detected in the SOAR data.

\section{CONCLUSIONS}

We performed follow-up observations on the hot DA white dwarf star HE 1017-1352. This object was reported as variable by Kurtz et al. (2013), following the theoretical predictions from Shibahashi (2005, 2007). We observed HE 1017-1352 with the SOAR telescope for a total of $16.76 \mathrm{~h}$ on three separate nights at the beginning of 2020. From the FT corresponding to the combination of the three nights, we found three periods of 508.121, 556.802, and $605.310 \mathrm{~s}$, with significant amplitudes above the detection limit. These periods are compatible with low harmonic degree $\mathrm{g}$ modes, with radial orders $k \gtrsim 9$. A low-amplitude mode with a period of $869.358 \mathrm{~s}$ is present, also compatible with a low $\ell$ mode, but for radial order $k \gtrsim 17$. The period at $605.310 \mathrm{~s}$ is in agreement with the peak at $614 \mathrm{~s}$ obtained from the WHT data and reported by Kurtz et al. (2013), within their uncertainties related to the FWHM of the peak of $280 \mu \mathrm{Hz}$. In addition, we detected a long period of $1.52 \mathrm{~h}$, which is compatible with a rotation period. Evidence of this long period can also be found in the observations from the TESS.

Our observations confirm the pulsational nature of HE 1017-1352 and thus the existence of the hot DAV class of pulsating stars. This opens the possibility to study the interior of these objects through asteroseismology. In addition, the confirmation of pulsations in hot DA stars is another evidence for the existence of white dwarfs with very thin hydrogen envelopes (Romero et al. 2012, 2019a), which are possibly a product of 


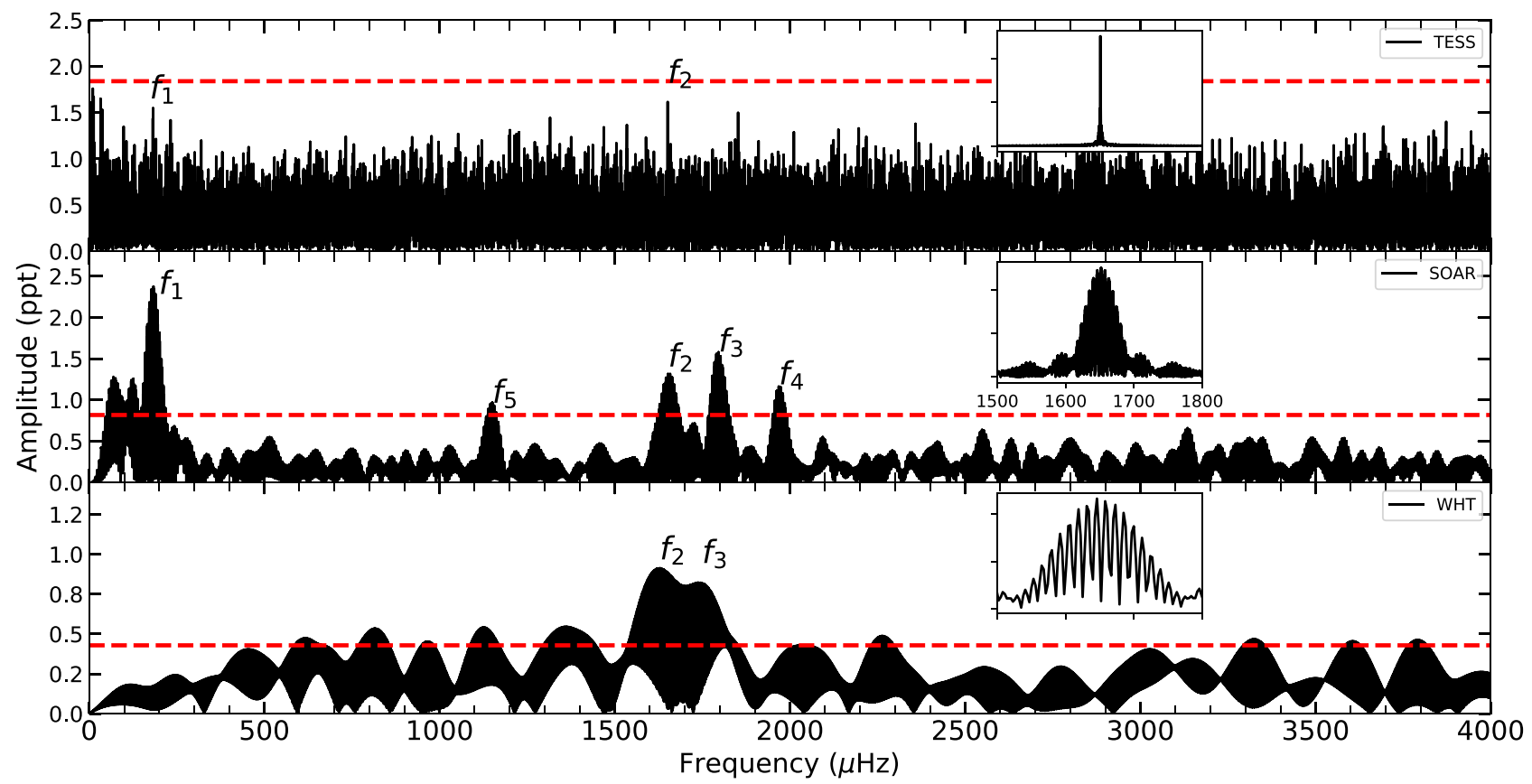

Figure 3. FT for HE 1017-1352 based on observations with three different instruments. Observations from TESS (top) span for $24 \mathrm{~d}$ in sector 9 . The FT for SOAR telescope (middle) was computed from the concatenation of the three nights presented in the previous section. The FT for the WHT (bottom) was computed from the original data considering the concatenation of the two nights. The FAP (1/1000) detection limit (dashed line) was computed using random shuffling of the data. The identification for each peak is indicated (see Table 3 for details). The spectral window for each case is depicted as an inset plot, with a full width at half-maximum (FWHM) of $1 \mu \mathrm{Hz}$ for TESS, $68 \mu \mathrm{Hz}$ for SOAR telescope, and $280 \mu \mathrm{Hz}$ for WHT. The $x$-axis is in $\mu \mathrm{Hz}$ and all inset plots are in the same scale as in the middle inset plot.

Table 3. Frequency, amplitude, and periods from observations with SOAR telescope (columns 1 to 3), TESS (columns 4 to 6), and WHT (columns 7 to 9), corresponding to the FT shown in Fig. 3. The quoted uncertainties correspond to the internal uncertainties. The value for the detection limit with 99.9 per cent confidence is indicated in each case in the first row. The last column shows the identification of the mode.

\begin{tabular}{|c|c|c|c|c|c|c|c|c|c|}
\hline Frequency $(\mu \mathrm{Hz})$ & $\begin{array}{l}\text { Amplitude } \\
\text { (ppt) }\end{array}$ & $\Pi(\mathrm{s})$ & $\begin{array}{c}\text { Frequency } \\
\quad(\mu \mathrm{Hz})\end{array}$ & $\begin{array}{l}\text { Amplitude } \\
\text { (ppt) }\end{array}$ & $\Pi(\mathrm{s})$ & $\begin{array}{l}\text { Frequency } \\
\quad(\mu \mathrm{Hz})\end{array}$ & $\begin{array}{l}\text { Amplitude } \\
\text { (ppt) }\end{array}$ & П (s) & ID \\
\hline \multicolumn{3}{|c|}{ SOAR $(0.8 \mathrm{ppt})$} & \multicolumn{3}{|c|}{ TESS (1.8 ppt) } & \multicolumn{4}{|c|}{ WHT (0.429 ppt) } \\
\hline $1652.046 \pm 0.005$ & $1.4 \pm 0.1$ & $605.310 \pm 0.002$ & $1650.90 \pm 0.07$ & $1.6 \pm 0.3$ & $605.73 \pm 0.02$ & $1628.27 \pm 0.04$ & $0.92 \pm 0.07$ & $614.15 \pm 0.02$ & $f_{2}$ \\
\hline $1795.970 \pm 0.004$ & $1.6 \pm 0.2$ & $556.802 \pm 0.002$ & - & - & - & $1748.87 \pm 0.05$ & $0.91 \pm 0.08$ & $571.80 \pm 0.02$ & $f_{3}$ \\
\hline $1968.034 \pm 0.007$ & $1.1 \pm 0.1$ & $508.121 \pm 0.002$ & - & - & - & - & - & - & $f_{4}$ \\
\hline $1150.274 \pm 0.008$ & $1.0 \pm 0.2$ & $869.358 \pm 0.006$ & - & - & - & - & - & - & $f_{5}$ \\
\hline
\end{tabular}

non-canonical formation channels, as close binary evolution and mergers.

\section{ACKNOWLEDGEMENTS}

We thank the anonymous referee for the useful comments and suggestions. ADR and SOK acknowledge financial support from CNPq and PRONEX-FAPERGS/CNPq (Brazil). LAA and LF acknowledge financial support from $\mathrm{CNPq}$ (Brazil). Based on observations at the Southern Astrophysical Research (SOAR) telescope, which is a joint project of MCTIC-Brazil, NOAOUS, the University of North Carolina at Chapel Hill (UNC), and Michigan State University (MSU), and processed using the IRAF package, developed by the Association of Universities for Research in Astronomy, Inc. This paper includes data collected with the TESS mission, obtained from the MAST data archive at the Space Telescope Science Institute (STScI). Funding for the TESS mission is provided by the NASA Explorer Program. This work has made use of data from the European Space Agency (ESA) mission Gaia (https://www.cosmos.esa.int/gaia), processed by the Gaia Data Processing and Analysis Consortium (DPAC, https: //www.cosmos.esa.int/web/gaia/dpac/consortium). Funding for the DPAC has been provided by national institutions, in particular the institutions participating in the Gaia Multilateral Agreement. This research has also made use of the NASA Astrophysics Data System.

\section{REFERENCES}

Althaus L. G., Panei J. A., Miller B. M. M., García-Berro E., Córsico A.

H., Romero A. D., Kepler S. O., Rohrmann R. D., 2009, ApJ, 704, 1605 Bergeron P., Wesemael F., Beauchamp A., 1995, PASP, 107, 1047

Bognár Z. et al., 2020, preprint (arXiv:2003.11481)

Fontaine G., Wesemael F., 1987, in Philip A. G. D., Hayes D. S., Liebert J. W., eds, IAU Colloq. 95: Second Conference on Faint Blue Stars. Davis Press, New York, p. 319

Gianninas A., Bergeron P., Ruiz M. T., 2011, ApJ, 743, 138

Kepler S. O. et al., 2016, MNRAS, 455, 3413 
Kepler S. O., Romero A. D., Pelisoli I., Ourique G., 2017, Int. J. Mod. Phys. Conf. Ser., 45, 1760023

Koester D., Kepler S. O., 2015, A\&A, 583, A86

Koester D., Voss B., Napiwotzki R., Christlieb N., Homeier D., Lisker T., Reimers D., Heber U., 2009, A\&A, 505, 441

Kurtz D. W., Shibahashi H., Dhillon V. S., Marsh T. R., Littlefair S. P., 2008, MNRAS, 389, 1771

Kurtz D. W., Shibahashi H., Dhillon V. S., Marsh T. R., Littlefair S. P., Copperwheat C. M., Gänsicke B. T., Parsons S. G., 2013, MNRAS, 432, 1632

Kurtz D. W., Shibahashi H., Murphy S. J., Bedding T. R., Bowman D. M., 2015, MNRAS, 450, 3015

Lenz P., Breger M., 2004, in Zverko J., Ziznovsky J., Adelman S. J., Weiss W. W., eds, Proc. IAU Symp. 224, The A-Star Puzzle. Cambridge Univ. Press, Cambridge, p. 786

Robinson E. L., Kepler S. O., Nather R. E., 1982, ApJ, 259, 219

Romero A. D., Istrate A. G., 2019, in Tovmassian G. H., Gansicke B. T., eds, Compact White Dwarf Binaries. p. 13

Romero A. D., Córsico A. H., Althaus L. G., Kepler S. O., Castanheira B. G., Miller Bertolami M. M., 2012, MNRAS, 420, 1462
Romero A. D., Kepler S. O., Joyce S. R. G., Lauffer G. R., Córsico A. H., 2019a, MNRAS, 484, 2711

Romero A. D. et al., 2019b, MNRAS, 490, 1803

Shibahashi H., 2005, in Alecian G., Richard O., Vauclair S., eds, EAS Publ. Ser. Vol. 17, Element Stratification in Stars: 40 Years of Atomic Diffusion. Cambridge Univ. Press, Cambridge, p. 143

Shibahashi H., 2007, in Stancliffe R. J., Houdek G., Martin R. G., Tout C. A., eds, AIP Conf. Proc. Vol. 948, Unsolved Problems in Stellar Physics: A Conference in Honor of Douglas Gough. Am. Inst. Phys., New York, p. 35

Tassoul M., 1980, ApJS, 43, 469

Vanderbosch Z. P., Winget K. I., Winget D. E., 2018, 21th European White Dwarf Workshop. Austin, Texas

Winget D. E., van Horn H. M., Tassoul M., Fontaine G., Hansen C. J., Carroll B. W., 1982, ApJ, 252, L65

This paper has been typeset from a $\mathrm{T}_{\mathrm{E}} \mathrm{X} / \mathrm{L} \mathrm{T} \mathrm{E} \mathrm{X}$ file prepared by the author. 